\title{
Multiple Solutions for Second-Order Sturm-Liouville Boundary Value Problems with Subquadratic Potentials at Zero
}

\author{
Dan Liu, Xuejun Zhang, and Mingliang Song $(\mathbb{D}$ \\ Mathematics and Information Technology School, Jiangsu Second Normal University, Nanjing 210013, China \\ Correspondence should be addressed to Mingliang Song; mlsong2004@163.com
}

Received 19 April 2021; Accepted 30 July 2021; Published 15 September 2021

Academic Editor: Nan-Jing Huang

Copyright (c) 2021 Dan Liu et al. This is an open access article distributed under the Creative Commons Attribution License, which permits unrestricted use, distribution, and reproduction in any medium, provided the original work is properly cited.

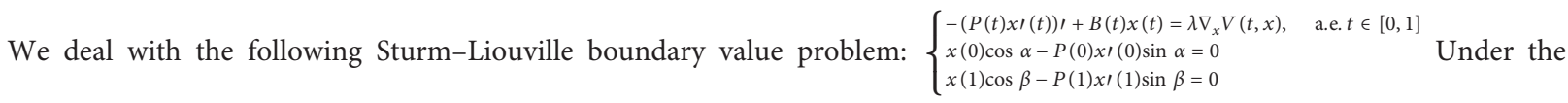
subquadratic condition at zero, we obtain the existence of two nontrivial solutions and infinitely many solutions by means of the linking theorem of Schechter and the symmetric mountain pass theorem of Kajikiya. Applying the results to Sturm-Liouville equations satisfying the mixed boundary value conditions or the Neumann boundary value conditions, we obtain some new theorems and give some examples to illustrate the validity of our results.

\section{Introduction and Main Results}

In this paper, we consider the Sturm-Liouville boundary value problem for second-order Hamiltonian systems:

$$
\left\{\begin{array}{l}
-\left(P(t) x^{\prime}(t)\right)^{\prime}+B(t) x(t)=\lambda \nabla_{x} V(t, x), \quad \text { a.e. } t \in[0,1] \\
x(0) \cos \alpha-P(0) x^{\prime}(0) \sin \alpha=0, \\
x(1) \cos \beta-P(1) x^{\prime}(1) \sin \beta=0,
\end{array}\right.
$$

where $\lambda>0, \alpha \in[0, \pi), \beta \in(0, \pi], \quad B(t) \in L^{\infty}\left([0,1], \mathscr{L}_{s}\right.$ $\left.\left(\mathbf{R}^{n}\right)\right)=\left\{B(t)=\left(b_{j k}\right)_{n \times n} \mid b_{j k} \quad(t)=b_{k j}(t), t \in[0,1], b_{j k}(t)\right.$ $\left.\in L^{\infty}([0,1])\right\}, P(t) \in C^{1}\left([(0,1)], \mathscr{L}_{s}\left(\mathbf{R}^{n}\right)\right)$ with $P(t)$ being positive definite for $t \in[0,1]$, and $\nabla_{x} V(t, x)$ denotes the gradient of $V(t, x)$ for $x \in \mathbf{R}^{n}$. We suppose that $V:[0,1] \times$ $\mathbf{R}^{n} \longrightarrow \mathbf{R}$ satisfies the following:

$\left(V_{0}\right) V(t, x)$ is measurable in $t$ for every $x \in \mathbf{R}^{n}$ and continuously differentiable in $x$ for a.e. $t \in[0,1]$. Moreover, if $n \geq 2$, then there exist $C_{0}>0$ and $\gamma>0$ such that

$$
\left|\nabla_{x} V(t, x)\right| \leq C_{0}\left(1+|x|^{\gamma}\right), \quad \forall x \in \mathbf{R}^{n} \text {, a.e. } t \in[0,1] \text {. }
$$

If $n=1$, then $\nabla_{x} V(t, x)=f(t, x):[0,1] \times \mathbf{R} \longrightarrow \mathbf{R}$ is a $L^{1}$-Carathéodory function.

Noticing that when $n=1, \alpha=0, \beta=\pi / 2$ or $n=1, \alpha=\beta=\pi / 2$, problem (1) becomes the mixed boundary value problem for Sturm-Liouville equations:

$$
\left\{\begin{array}{l}
-\left(P(t) x^{\prime}(t)\right)^{\prime}+B(t) x(t)=\lambda f(t, x), \quad \text { a.e. } t \in[0,1] \\
x(0)=x^{\prime}(1)=0
\end{array}\right.
$$

or the Neumann boundary value problem for Sturm-Liouville equations:

$$
\left\{\begin{array}{l}
-\left(P(t) x^{\prime}(t)\right)^{\prime}+B(t) x(t)=\lambda f(t, x), \quad \text { a.e. } t \in[0,1] \\
x^{\prime}(0)=x^{\prime}(1)=0
\end{array}\right.
$$

which show that problems (3) and (4) are two special cases of (1).

Recently, the existence of two nontrivial solutions and infinitely many solutions for problems (3) and (4) have been extensively studied and some useful results have been 
obtained (see [1-12]). Their tools are based on some abstract multiple theorems established by Bonanno and Candito [13], Bonanno and D'Aguì [6], and Ricceri [14]. However, linear term $B(t) \geq 0$ is necessary in the conditions of theorems discussing the existence of two nontrivial solutions for problem (3) in $[1,2,9,10]$. $B(t) \geq 0$ and $\lambda \in\left(\lambda_{1}, \lambda_{2}\right) \subset(0,+\infty)$ are necessary conditions in the existence theorems of infinitely many solutions for problem (3) in $[3,4]$. For problem (4), $B(t) \equiv M \geq 0$ and $\lambda=1$ are necessary in the existence theorems of two nontrivial solutions in $[11,12]$. $B(t) \in C\left([0,1], \mathbf{R}^{+} \backslash\{0\}\right)$ and $\lambda \in\left(\lambda_{1}, \lambda_{2}\right) \subset(0,+\infty)$ are also necessary in the existence of infinitely many solutions for problem (4) in [5]. In this paper, we are interested in the assumption without $B(t) \geq 0$ and $\lambda \in\left(\lambda_{1}, \lambda_{2}\right) \subset(0,+\infty)$ assumptions.

Moreover, with the aid of variational methods, the multiplicity of periodic solutions for Hamiltonian systems has also been extensively investigated in many papers and books (see [15-23] and the references therein). In particular, in [24], using the linking theorem of Schechter $[19,20]$, Bonanno et al. have discussed the existence of two nontrivial solutions for second-order Hamiltonian systems with subquadratic potentials at zero. For infinitely many solutions of subquadratic second-order Hamiltonian systems, Zou and Li [22] obtained two existence theorems via the minimax technique of Fei [25], and Zhang and Liu [23] obtained an existence theorem via the variant fountain theorem of Zou [26]. After that, by the symmetric mountain pass theorem of Kajikiya [27], Yi and Tang [21] obtained an existence theorem, which unifies and improves upon theorems of Zou and Li and Zhang and Liu [22, 23].

Inspired by the ideas of Ye and Tang and Bonanno et al. $[21,24]$, in this paper, we shall study the existence of two nontrivial solutions and infinitely many solutions for problem (1), where $V(t, x)$ is subquadratic at zero. Based on the index theory of Dong $[15,16]$, the linking theorem of Schechter $[19,20]$, and the symmetric mountain pass theorem of Kajikiya [27], we will prove the existence of two nontrivial solutions and infinitely many solutions. Applying the results to problems (3) and (4), we obtain some new theorems. Meanwhile, some examples of problems (3) and (4) are given to illustrate the validity of our result and point out that linear terms $B(t)<0$ and $\lambda \in(0,+\infty)$ are allowed, which show that our results are also new even in the cases of (3) and (4).

Next we use the index $\left(i_{\alpha, \beta}^{P}(B), \nu_{\alpha, \beta}^{P}(B)\right) \in \mathbf{N} \times \mathbf{N}$ defined in [15] (see Section 2) for all $B(t) \in L^{\infty}\left([0,1], \mathscr{L}_{s}\left(\mathbf{R}^{n}\right)\right)$ to give our main results.

Theorem 1. Assume that $V:[0,1] \times \mathbf{R}^{n} \longrightarrow \mathbf{R}$ satisfies $\left(V_{0}\right)$ and

$$
\begin{aligned}
& \left(V_{1}\right) i_{\alpha, \beta}^{P}(B)=v_{\alpha, \beta}^{P}(B)=0 . \\
& \left(V_{2}\right) \text { There exist } \eta>0,0<\vartheta<2 \text { and a function } \\
& g(t) \in L^{1}\left([0,1], \mathbf{R}^{+}\right) \text {such that }
\end{aligned}
$$$$
V(t, x) \leq g(t)|x|^{9}, \quad|x| \leq \eta, x \in \mathbf{R}^{n} .
$$

$\left(V_{3}\right)$ There exists a constant $\kappa_{1}>\kappa_{0}=\delta_{0} \eta^{9-2}\|g\|_{L^{1}}$ such that

$$
\begin{aligned}
& \liminf _{c \longrightarrow 0} \frac{\int_{0}^{1} V(t, c \varphi) \mathrm{d} t}{c^{2}\|\varphi\|_{L^{2}}^{2}}>\kappa_{1} \lambda_{0}, \\
& \liminf _{c \longrightarrow+\infty} \frac{\int_{0}^{1} V(t, c \varphi) \mathrm{d} t}{c^{2}\|\varphi\|_{L^{2}}^{2}}>\kappa_{1} \lambda_{0},
\end{aligned}
$$

where $\varphi$ is an eigenfunction of the operator $\Lambda_{B}$ corresponding to the first eigenvalue $\lambda_{0}$ and $\delta_{0}>0$ is a constant such that

$$
\|x\|_{\infty}^{2} \leq \delta_{0}\left[a(x, x)+\int_{0}^{1}(B(t) x, x) \mathrm{d} t\right], \quad x \in Z=D\left(|\Lambda|^{1 / 2}\right) .
$$

Then, problem (1) has two nontrivial solutions for almost all $\lambda \in\left(1 / 2 \kappa_{1}, 1 / 2 \kappa_{0}\right)$.

Remark 1. In Theorem 1, the operator $\Lambda_{B}$ is defined as $\left(\Lambda_{B} x\right)(t)=-\left(P(t) x^{\prime}\right) \prime(t)+B(t) x(t)(\forall x \in D(\Lambda)) . D(\Lambda)$ and $a(x, x)$ are defined by (13) and (15) in Section 2, respectively. The existence of $\delta_{0}$ is proved in (33) of Section 3.

Theorem 2. Assume that $V:[0,1] \times \mathbf{R}^{n} \longrightarrow \mathbf{R}$ satisfies $\left(V_{0}\right)$ and

$$
\begin{aligned}
& \left(V_{4}\right) V(t, x) \text { is even in } x \text { for a.e. } t \in[0,1] . \\
& \left(V_{5}\right) V(t, 0)=0 \text { for a.e. } t \in[0,1] \text { and } \\
& \lim _{x \mid \longrightarrow 0} \mid \frac{V(t, x)}{|x|^{2}}=+\infty \text { uniformly for a.e.t } \in[0,1] .
\end{aligned}
$$

Then, problem (1) has infinitely many small-energy solutions for each $\lambda>0$.

Remark 2. In Theorem 2, we do not need any restrictions on $i_{\alpha, \beta}^{P}(B)$ and $v_{\alpha, \beta}^{P}(B)$, which means that $i_{\alpha, \beta}^{P}(B) \neq 0, v_{\alpha, \beta}^{P}(B)=0$ or $i_{\alpha, \beta}^{P}(B) \neq 0, v_{\alpha, \beta}^{P}(B) \neq 0 \quad$ or $i_{\alpha, \beta}^{P}(B)=0, v_{\alpha, \beta}^{P}(B) \neq 0$ or $i_{\alpha, \beta}^{P}(B)=\nu_{\alpha, \beta}^{P}(B) \stackrel{\alpha, \beta}{=} 0$ are allowed.

The paper is arranged as follows. In Section 2, we recall some useful conclusions of index theory for linear secondorder Hamiltonian systems from $[15,16]$ and verify that problem (1) possesses a variational construction in $Z$. In Section 3, using the linking theorem of Schechter $[19,20]$ and the symmetric mountain pass theorem of Kajikiya [27], we prove Theorems 1 and 2. In Section 4, we investigate their applications to Sturm-Liouville equations with the mixed boundary value conditions and the Neumann boundary value conditions. Meanwhile, some examples are given to show that our results are also new even in the cases of problems (3) and (4).

\section{Preliminaries and Variational Setting}

For the reader's convenience, we first recall some useful conclusions of index theory for linear second-order Hamiltonian systems given in $[15,16]$, respectively. 
Index theory in $[15,16]$ deals with a classification of $L^{\infty}\left([0,1], \mathscr{L}_{s}\left(\mathbf{R}^{n}\right)\right)$ associated with the following Lagrangian systems:

$$
\begin{array}{r}
-\left(P(t) x^{\prime}\right)^{\prime}+B(t) x=0, \\
x(0) \cos \alpha-P(0) x^{\prime}(0) \sin \alpha=0, \\
x(1) \cos \beta-P(1) x^{\prime}(1) \sin \beta=0,
\end{array}
$$

where $\quad B(t) \in L^{\infty}\left([0,1], \mathscr{L}_{s}\left(\mathbf{R}^{n}\right)\right), \quad P(t) \in C^{1}\left([0,1], \mathscr{L}_{s}\right.$ $\left(\mathbf{R}^{n}\right)$ ) with $P(t)$ being positive definite for $t \in[0,1]$, and $\alpha \in[0, \pi), \beta \in(0, \pi]$.

Let $X=L^{2}\left([0,1], \mathbf{R}^{n}\right)$. Set $(\Lambda x)(t)=-\left(P(t) x^{\prime}\right)^{\prime}(t)$ with $D(\Lambda)=\left\{x \in H^{2}\left([0,1], \mathbf{R}^{n}\right): x\right.$ satisfies $(11)$ and $\left.(12)\right\}$.
By Section 2.3 in [15], we know that $\Lambda$ is self-adjoint and $\sigma(\Lambda)=\sigma_{d}(\Lambda)=\{\lambda \in \mathbf{R}: \lambda$ belongs to the point spectrum of $\Lambda\}$

is bounded from below. We define a bilinear form as follows:

$$
\begin{aligned}
a(x, y)= & \int_{0}^{1}\left(P(t) x^{\prime}(t), y^{\prime}(t)\right) \mathrm{d} t-(x(1), y(1)) \gamma(\beta) \\
& +(x(0), y(0)) \gamma(\alpha),
\end{aligned}
$$

for all $x, y \in Z$, where $(\cdot, \cdot)$ is the usual inner product in $\mathbf{R}^{n}$, $\gamma(s)=\cot s$ as $s \in(0, \pi), \gamma(s)=0$ as $s=0$ or $s=\pi$, and

$$
Z= \begin{cases}\left\{x \in H^{1}\left([0,1], \mathbf{R}^{n}\right): x(1)=0\right\}, & \alpha=0, \beta \in(0, \pi) ; \\ \left\{x \in H^{1}\left([0,1], \mathbf{R}^{n}\right): x(0)=0\right\}, & \alpha \in(0, \pi), \beta=\pi ; \\ \left\{x \in H^{1}\left([0,1], \mathbf{R}^{n}\right): x^{\prime}(0)=x^{\prime}(1)=0\right\}, & \alpha=\beta=\frac{\pi}{2} ; \\ \left\{x \in H^{1}\left([0,1], \mathbf{R}^{n}\right): x(0)=x^{\prime}(1)=0\right\}, & \alpha=0, \beta=\frac{\pi}{2} ; \\ \left\{x \in H^{1}\left([0,1], \mathbf{R}^{n}\right): x(1)=x(0)=0\right\}, & \alpha=0, \beta=\pi ; \\ H^{1}\left([0,1], \mathbf{R}^{n}\right), & \alpha, \beta \in(0, \pi) .\end{cases}
$$

Similar to the proof of Proposition 1.17 in [28], we know that $Z$ is a Hilbert space and $Z=D\left(|\Lambda|^{1 / 2}\right)$. By Lemma 2.3.1 of [15], we find that $Z=D\left(|\Lambda|^{1 / 2}\right)$ can be equipped with the equivalent norm

$$
\|x\|_{Z}=\left(\int_{0}^{1}\left[\left|x^{\prime}(t)\right|^{2}+|x(t)|^{2}\right] \mathrm{d} t\right)^{1 / 2},
$$

for each $x \in Z$. Clearly, the embeddings $Z \longrightarrow L^{2}=X$ and $Z \longrightarrow L^{\infty}$ are compact. For any $B(t) \in L^{\infty}\left([0,1], \mathscr{L}_{s}\left(\mathbf{R}^{n}\right)\right)$, we define

$$
\psi_{\alpha, \beta}^{P, B}(x, y)=a(x, y)+\int_{0}^{1}(B(t) x(t), y(t)) \mathrm{d} t,
$$

for all $x, y \in Z$.

Proposition 1 (see [15], Proposition 2.1.1]). For any $B(t) \in L^{\infty}\left([0,1], \mathscr{L}_{s}\left(\mathbf{R}^{n}\right)\right)$, the space $Z$ has a $\psi_{\alpha, \beta}^{P, B}$-orthogonal decomposition

$$
Z=Z^{+}(B) \oplus Z^{0}(B) \oplus Z^{-}(B),
$$

such that $\psi_{\alpha, \beta}^{P, B}$ is positive definite, null, and negative definite on $Z^{+}(B), Z^{0}(B)$, and $Z^{-}(B)$, respectively. Moreover, $Z^{0}(B)$ and $Z^{-}(B)$ are finitely dimensional.

Definition 1 (see [15], Definition 2.3.2]). For any $B(t) \in L^{\infty}\left([0,1], \mathscr{L}_{s}\left(\mathbf{R}^{n}\right)\right)$, we define

$$
\begin{aligned}
& v_{\alpha, \beta}^{P}(B)=\operatorname{dimker}(\Lambda+B), \\
& i_{\alpha, \beta}^{P}(B)=\sum_{\lambda>0} v_{\alpha, \beta}^{P}\left(B+\lambda I_{n}\right) .
\end{aligned}
$$

Proposition 2 (see [16], Definition 7.2.1 and Proposition 7.2.2 (1)]). For any $B \in L^{\infty}\left([0,1], \mathscr{L}_{s}\left(\mathbf{R}^{n}\right)\right)$, we have that $Z^{0}(B)$ is the solution subspace of systems (10)-(12), and

$$
\begin{aligned}
& v_{\alpha, \beta}^{P}(B)=\operatorname{dim} Z^{0}(B), \\
& i_{\alpha, \beta}^{P}(B)=\operatorname{dim} Z^{-}(B) .
\end{aligned}
$$


We call $\nu_{\alpha, \beta}^{P}(B)$ and $i_{\alpha, \beta}^{P}(B)$ the nullity and index of $B$ with respect to the bilinear form $\psi_{\alpha, \beta}^{P, B}(\cdot, \cdot)$.

Proposition 3 (see [29], Proposition 2.6 (5)]). For any $B \in L^{\infty}\left([(0,1)], \mathscr{L}_{s}\left(\mathbf{R}^{n}\right)\right)$, if $x \in Z$ satisfying $x=x_{1}+x_{2}$ with $x_{1} \in Z^{-}(B), x_{2} \in Z^{+}(B)$, then $\left(-\psi_{\alpha, \beta}^{P, B}\left(x_{1}, x_{1}\right)\right)^{1 / 2}+$ $\left(\psi_{\alpha, \beta}^{P, B}\left(x_{2}, x_{2}\right)\right)^{1 / 2}$ is an equivalent norm on $Z$.

Proposition 4 (see [15], Proposition 2.3.3 (3)]). For any $B_{1}, B_{2} \in L^{\infty}\left([0,1], \mathscr{L}_{s}\left(\mathbf{R}^{n}\right)\right)$ satisfying $B_{1}(t)>B_{2}(t)$ for all $t \in[0,1]$, we have $v_{\alpha, \beta}^{P}\left(B_{1}\right)+i_{\alpha, \beta}^{P}\left(B_{1}\right) \leq i_{\alpha, \beta}^{P}\left(B_{2}\right)$.

Remark 3. (see [16], Remark 7.1.2]) Let $P(t) \equiv I_{n}$. Then,

$$
\begin{aligned}
& v_{0, \pi / 2}^{I_{n}}\left(c I_{n}\right)=n \text { as } c=-\left(\frac{1}{2}+k\right) \pi^{2}, \\
& v_{0, \pi / 2}^{I_{n}}\left(c I_{n}\right)=0 \text { as } c \neq-\left(\frac{1}{2}+k\right)^{2} \pi^{2}, \quad \text { for } k \in N, \\
& i_{0, \pi / 2}^{I_{n}}\left(c I_{n}\right)=0 \text { as } c \geq-\frac{\pi^{2}}{4}, \\
& i_{0, \pi / 2}^{I_{n}}\left(c I_{n}\right)=(k+1) n \text { as } c \in\left(-\left(\frac{1}{2}+k+1\right) \pi^{2},-\left(\frac{1}{2}+k\right)^{2} \pi^{2},\right) ; \\
& v_{\pi / 2, \pi / 2}^{I_{n}}\left(c I_{n}\right)=n \text { as } c=-k^{2} \pi^{2}, \\
& v_{\pi / 2, \pi / 2}^{I_{n}}\left(c I_{n}\right)=0 \text { as } c \neq-k^{2} \pi^{2}, \quad \text { for } k \in N, \\
& i_{\pi / 2, \pi / 2}^{I_{n}}\left(c I_{n}\right)=0 \text { as } c \geq 0, \\
& i_{\pi / 2, \pi / 2}^{I_{n}}\left(c I_{n}\right)=(k+1) n \text { as } c \in\left(-(k+1)^{2} \pi^{2},-k^{2} \pi^{2}\right) .
\end{aligned}
$$

Next, let us consider the functional $I$ defined by

$$
I(x)=\frac{a(x, x)}{2}+\frac{1}{2} \int_{0}^{1}(B(t) x, x) \mathrm{d} t-\lambda \int_{0}^{1} V(t, x) \mathrm{d} t, \quad \forall x \in Z .
$$

From assumption $\left(V_{0}\right)$, using Theorem 1.4 [17], it is easy to check that $I$ is continuously differentiable and weakly lower semicontinuous on $Z$, and

$$
I^{\prime}(x) y=a(x, y)+\int_{0}^{1}(B(t) x, y) \mathrm{d} t-\lambda \int_{0}^{1}\left(\nabla_{x} V(t, x), y\right) \mathrm{d} t,
$$

for all $x, y \in Z$. If $I^{\prime}(x)=0$, then we have

$$
\begin{array}{r}
\int_{0}^{1}\left(P(t) x^{\prime}, y^{\prime}\right) \mathrm{d} t-(x(1), y(1)) \gamma(\beta)+(x(0), y(0)) \gamma(\alpha) \\
+\int_{0}^{1}(B(t) x, y) \mathrm{d} t-\lambda \int_{0}^{1}\left(\nabla_{x} V(t, x), y\right) \mathrm{d} t=0,
\end{array}
$$

for all $y \in H^{1}\left([0,1], \mathbf{R}^{n}\right)$. Choosing $y \in H^{1}\left([0,1], \mathbf{R}^{n}\right)$ satisfying $y(0)=y(1)=0$, one has

$$
\int_{0}^{1}\left(P(t) x^{\prime}, y^{\prime}\right) \mathrm{d} t+\int_{0}^{1}(B(t) x, y) \mathrm{d} t-\lambda \int_{0}^{1}\left(\nabla_{x} V(t, x), y\right) \mathrm{d} t=0 .
$$

Put $e(t)=-P(t) x^{\prime}+\int_{0}^{t} B(s) x(s) \mathrm{d} s-\lambda \int_{0}^{t} \nabla_{x} V(s, x(s))$ $\mathrm{d} s-c$ such that $\int_{0}^{1} e(t) \mathrm{d} t=0$. Let $y(t)=\int_{0}^{t} e(s) \mathrm{d} s$. Then, we have $\int_{0}^{1}|e(t)|^{2} \mathrm{~d} t=0$ and $e(t)=0$ for a.e. $t \in[0,1]$, which imply that $x(t)$ satisfies $x \in H^{2}\left([0,1], \mathbf{R}^{n}\right)$ and

$$
-\left(P(t) x^{\prime}(t)\right)+B(t) x(t)-\lambda \nabla_{x} V(t, x)=0 .
$$

Form (25), it follows that

$$
\begin{aligned}
0= & \int_{0}^{1}\left(P(t) x^{\prime}, y^{\prime}\right) \mathrm{d} t-(x(1), y(1)) \gamma(\beta)+(x(0), y(0)) \gamma(\alpha) \\
& +\int_{0}^{1}\left(\left(P(t) x^{\prime}(t)\right) \prime, y\right) \mathrm{d} t \\
= & \left(P(1) x^{\prime}(1), y(1)\right)-\left(P(0) x^{\prime}(0), y(0)\right) \\
& -(x(1), y(1)) \gamma(\beta)+(x(0), y(0)) \gamma(\alpha),
\end{aligned}
$$

for all $y \in H^{1}\left([0,1], \mathbf{R}^{n}\right)$. This shows that $x(t)$ satisfies (11)-(12), which means that the critical points of $I$ correspond to the classical solutions of problem (1).

\section{Proofs of the Theorems}

In order to prove Theorem 1, we recall some results of linking given by Schechter $[19,20]$.

Let $E$ be a reflexive Banach space with norm $\|\cdot\|$. The set $\Phi=\{\Gamma(t): \Gamma(t) \in C(E \times[0,1], E)\}$ is to have the following properties:

(i) $\Gamma(0)=I$, the identity map.

(ii) For each $t \in[0,1), \Gamma(t)$ is a homeomorphism of $E$ onto itself and $\Gamma^{-1}(t) \in C(E \times[0,1], E)$.

(iii) $\Gamma(1) E$ is a single point $u_{\Gamma} \in E$ and $\Gamma(t) A$ converges uniformly to $u_{\Gamma}$ as $t \longrightarrow 1$ for each bounded set $A \subset E$.

(iv) For each $t_{0} \in[0,1)$ and each bounded set $A \subset E$, one has

$$
\sup _{0 \leq t \leq t_{0}, u \in A}\left\{\|\Gamma(t) u\|+\left\|\Gamma^{-1}(t) u\right\|\right\}<+\infty .
$$


Definition 2 (see [19], Definition 3.2]). A subset $A \subset E$ links $B \subset E[\mathrm{hm}]$ if $A \cap B=\varnothing$, and for each $\Gamma(t) \in \Phi$, there is a $t \in(0,1]$ such that $\Gamma(t) A \cap B \neq \varnothing$.

Theorem 3 (see [20], Theorem 4.1]). Let $\mathscr{F}, \mathscr{J} \in C^{1}(E, \mathbf{R})$ be bounded on bounded sets and let

$$
G_{\mu}(u)=\mu \mathscr{F}(u)-\mathscr{J}(u), \quad \mu \in \mathscr{I},
$$

where $\mathscr{I}$ is an open interval contained in $(0,+\infty)$. Assume that $G_{\mu}$ satisfies

$\left(H_{1}\right) \mathscr{F}(u) \geq 0$ for all $u \in E$ and $\mathscr{F}(u)+|\mathscr{F}(u)| \longrightarrow+$ $\infty$ as $\|u\| \longrightarrow+\infty$.

$\left(\mathrm{H}_{2}\right)$ There are sets $A, B \subset E$ such that $A$ links $B[\mathrm{hm}]$ and

$$
a_{0}=\sup _{A} G_{\mu} \leq b_{0}=\inf _{B} G_{\mu}
$$

for each $\mu \in \mathcal{I}$.

$\left(H_{3}\right) a(\mu)=\inf _{\Gamma \in \Phi} \sup _{0 \leq s \leq 1, u \in A} G_{\mu}(\Gamma(s) u)$ is finite for each $\mu \in \mathscr{I}$.

Then, for almost all $\mu \in \mathscr{I}$, there exists a bounded sequence $u_{k}(\mu) \in E$ such that

$$
\begin{aligned}
& \left\|G_{\mu}^{\prime}\left(u_{k}\right)\right\| \longrightarrow 0, \\
& G_{\mu}\left(u_{k}\right) \longrightarrow a(\mu) \text { as } k \longrightarrow+\infty .
\end{aligned}
$$

Lemma 1 (see [19], Example 1 of Section 3.5]). Let $B$ be an open set in $E$ and let $A$ consist of two points $e_{1}, e_{2}$ with $e_{1} \in B$ and $e_{2} \notin \bar{B}$. Then, $A$ links $\partial B[\mathrm{hm}]$. $\partial B$ links $A[\mathrm{hm}]$ as well if $\partial B$ is bounded.

Proof of Theorem 1. From $i_{\alpha, \beta}^{P}(B)=v_{\alpha, \beta}^{P}(B)=0$ of $\left(V_{1}\right)$, by Propositions 1 and 2 and Definition 1 , we know that $Z=$ $Z^{+}(B)$ and $\lambda_{0}=\min \sigma_{d}\left(\Lambda_{B}\right)>0$. By Proposition 3, we can see that $\left(\psi_{\alpha, \beta}^{P, B}(\cdot, \cdot)\right)^{1 / 2}=\|\cdot\|$ is an equivalent norm on $Z$. Since the embedding $Z \longrightarrow L^{\infty}$ is compact, there is $\delta_{0}>0$ such that

$$
\begin{aligned}
\|x\|_{\infty}^{2} & \leq \delta_{0}\left[a(x, x)+\int_{0}^{1}(B(t) x, x) \mathrm{d} t\right] \\
& =\delta_{0} \psi_{\alpha, \beta}^{P, B}(x, x)=\delta_{0}\|x\|^{2}, \quad x \in Z .
\end{aligned}
$$

Set $\varphi$ is an eigenfunction of the operator $\Lambda_{B}$ corresponding to the first eigenvalue $\lambda_{0}, \quad \mu=1 / \lambda$, $\mathscr{F}(x)=1 / 2 \psi_{\alpha, \beta}^{P, B}(x, x)=1 / 2\|x\|^{2}, \mathscr{J}(x)=\int_{0}^{1} V(t, x) \mathrm{d} t$, and

$$
G_{\mu}(x)=\mu \mathscr{F}(x)-\mathscr{J}(x), \quad \forall x \in Z .
$$

Clearly, $\mathscr{F} \in C^{1}(E, \mathbf{R})$ is bounded on bounded sets, $\mathscr{J} \in C^{1}(Z, \mathbf{R})$, and $G_{\mu}^{\prime}(x)=0$ is equivalent to $I^{\prime}(x)=0$, which shows that the critical points of $G_{\mu}$ correspond to the solutions of problem (1). By $\left(V_{0}\right), \mathcal{J}$ is also bounded on bounded sets.

Fix $\mu \in\left(2 \kappa_{0}, 2 \kappa_{1}\right)$, put $r=\eta / \sqrt{\delta_{0}}$, and set

$$
\begin{aligned}
B_{r} & =\{x \in Z:\|x\| \leq r\}, \\
\partial B_{r} & =\{x \in Z:\|x\|=r\} .
\end{aligned}
$$

Let $\varepsilon>0$ be such that $2 \kappa_{0}<2 \kappa_{0}+\varepsilon<\mu<2 \kappa_{1}$. Then, for every $x \in \partial B_{r}$, we have

$$
\begin{aligned}
G_{\mu}(x) & \geq \frac{\mu}{2}\|x\|^{2}-\int_{0}^{1} g(t)|x(t)|^{9} \mathrm{~d} t \geq \frac{\mu \eta^{2}}{2 \delta_{0}}-\eta^{9}\|g\|_{L^{1}} \\
& =\frac{\mu \eta^{2}}{2 \delta_{0}}-\frac{\kappa_{0} \eta^{2}}{\delta_{0}}>\frac{\varepsilon \eta^{2}}{2 \delta_{0}}
\end{aligned}
$$

via $\left(V_{2}\right)$ and $\kappa_{0}=\delta_{0} \eta^{9-2}\|g\|_{L^{1}}$, which implies that

$$
b(\mu)=\inf _{x \in \partial B_{r}} G_{\mu}(x)>0 .
$$

For every $x \in B_{r}$, by $\left(V_{2}\right)$ and (33), one has

$$
\begin{aligned}
G_{\mu}(x) & \geq \frac{\mu}{2}\|x\|^{2}-\int_{0}^{1} g(t)|x(t)|^{9} \mathrm{~d} t \geq \frac{\mu}{2}\|x\|^{2}-\sqrt{\delta_{0}^{9}}\|x\|^{9}\|g\|_{L^{1}}, \\
d(\mu) & =\inf _{x \in B_{r}} G_{\mu}(x)>-\infty .
\end{aligned}
$$

Set $\quad \liminf _{c \longrightarrow 0} \int_{0}^{1} V(t, c \varphi) \mathrm{d} t / c^{2}\|\varphi\|_{L^{2}}^{2}=M_{0} . \quad$ Fix $\epsilon \in\left(0, M_{0}-\kappa_{1} \lambda_{0}\right)$; from (6), we know that there exists $\xi>0$ such that

$$
\frac{\int_{0}^{1} V(t, c \varphi) \mathrm{d} t}{c^{2}\|\varphi\|_{L^{2}}^{2}}>\kappa_{1} \lambda_{0}+\epsilon,
$$

for all $|c|<\xi$. Hence, for $|c|$ small enough, we have $c \varphi \in B_{r}$ and

$$
\begin{aligned}
G_{\mu}(c \varphi) & =\frac{\mu \lambda_{0} c^{2}}{2}\|\varphi\|_{L^{2}}^{2}-\int_{0}^{1} V(t, c \varphi) \mathrm{d} t \\
& \leq c^{2}\|\varphi\|_{L^{2}}^{2}\left(\kappa_{1} \lambda_{0}-\frac{\int_{0}^{1} V(t, c \varphi) \mathrm{d} t}{c^{2}\|\varphi\|_{L^{2}}^{2}}\right) \\
& \leq-\epsilon c^{2}\|\varphi\|_{L^{2}}^{2}<0,
\end{aligned}
$$

which shows that $0>\inf _{x \in B_{r}} G_{\mu}(x)=d(\mu)>-\infty$. There exists a minimizing sequence $\left\{x_{k}\right\} \subset B_{r}$ such that $G_{\mu}\left(x_{k}\right) \longrightarrow d(\mu)$ as $k \longrightarrow \infty$. Noticing that the embed$\operatorname{ding} Z \longrightarrow L^{\infty}$ is compact, one has a renamed subsequence such that $x_{k} \rightarrow x \in Z$ and $x_{k} \longrightarrow x \in L^{\infty}([0,1], \mathbf{R})$. Then, we have

$$
\begin{aligned}
& \mu \mathscr{F}\left(x_{k}\right) \longrightarrow d(\mu)+\int_{0}^{1} V(t, x) \mathrm{d} t, \\
& \mu \mathscr{F}(x) \leq \lim \inf \mu \mathscr{F}\left(x_{k}\right)=d(\mu)+\int_{0}^{1} V(t, x) \mathrm{d} t,
\end{aligned}
$$

as $k \longrightarrow \infty$. This shows that $G_{\mu}(x) \leq d(\mu)<0$ and $x \notin \partial B_{r}$. Hence, $x$ is in the interior $B_{r}$, and we have $G_{\mu}^{\prime}(x)=0$.

By $\mathscr{F}(x)=1 / 2\|x\|^{2}$, it is clear that $\left(H_{1}\right)$ holds. Set $\lim \inf _{c \longrightarrow+\infty} \int_{0}^{1} V(t, c \varphi) \mathrm{d} t / c^{2}\|\varphi\|_{L^{2}}^{2}=M_{1}$. Fix $\epsilon_{1} \in\left(0, M_{1}-\right.$ $\kappa_{1} \lambda_{0}$ ); from (7), we know that there exists $\xi_{1}>0$ such that 
$\int_{0}^{1} V(t, c \varphi) \mathrm{d} t / c^{2}\|\varphi\|_{L^{2}}^{2}>\kappa_{1} \lambda_{0}+\epsilon_{1}$ for all $|c|>\xi_{1}$. Hence, for $|c|$ large enough, we have $c \varphi \notin B_{r}$ and

$$
\begin{aligned}
G_{\mu}(c \varphi) & \leq c^{2}\|\varphi\|_{L^{2}}^{2}\left(\kappa_{1} \lambda_{0}-\frac{\int_{0}^{1} V(t, c \varphi) \mathrm{d} t}{c^{2}\|\varphi\|_{L^{2}}^{2}}\right) \\
& \leq-\epsilon_{1} c^{2}\|\varphi\|_{L^{2}}^{2}<0 .
\end{aligned}
$$

From (40) and (42), we can see that there are $c_{1}, c_{2}$ such that $c_{1} \varphi \in B_{r}, c_{1} \varphi \notin \partial B_{r}$ and $c_{2} \varphi \notin B_{r}$ with $G_{\mu}\left(c_{i} \varphi\right)<$ $0, i=1,2$. Set $A=\left\{c_{1} \varphi, c_{2} \varphi\right\}$ and $B=\partial B_{r}$. By Lemma 1 , we know that $A$ links $B[\mathrm{hm}]$. Clearly, $a_{0}=\sup _{\mathrm{A}} G_{\mu}<0$ $<b_{0}=\inf _{B} G_{\mu},\left(H_{2}\right)$ and $\left(H_{3}\right)$ holds. Using Theorem 3 and Definition 2, for almost all $\mu \in \mathscr{I}$, there exists a bounded sequence $y_{k}(\mu) \in Z$ such that

$$
\begin{aligned}
& G_{\mu}^{\prime}\left(y_{k}\right) \longrightarrow 0, \\
& G_{\mu}\left(y_{k}\right) \longrightarrow a(\mu)=\inf _{\Gamma \in \Phi} \sup _{0 \leq s \leq 1, u \in A} \\
& G_{\mu}(\Gamma(s) u) \geq b(\mu)>0, \text { as } k \longrightarrow+\infty .
\end{aligned}
$$

Since the embedding $Z \longrightarrow L^{\infty}$ is compact, there exists a renamed subsequence such that $y_{k} \rightarrow y \in Z$ and $y_{k} \longrightarrow y \in L^{\infty}([0,1], \mathbf{R})$. Since $G_{\mu}^{\prime}\left(y_{k}\right) \longrightarrow 0$, one has

$$
\begin{aligned}
& \mu\left\|y_{k}\right\|^{2}-\int_{0}^{1}\left(\nabla_{x} V\left(t, y_{k}\right), y_{k}\right) \mathrm{d} t \longrightarrow 0, \\
& \mu\left\|y_{k}\right\|^{2} \longrightarrow \int_{0}^{1}\left(\nabla_{x} V(t, y), y\right) \mathrm{d} t
\end{aligned}
$$

as $k \longrightarrow \infty$, which shows that $y_{k} \longrightarrow y \in Z$ and $G_{\mu}^{\prime}(y)=0$. Consequently, we have $G_{\mu}\left(y_{k}\right) \longrightarrow G_{\mu}(y)$ and $G_{\mu}(y)=$ $a(\mu) \geq b(\mu)>0$. Noticing that $\lambda=1 / \mu, G_{\mu}(x)<0, G_{\mu}(y)>0$, and $G_{\mu}^{\prime}(x)=G_{\mu}^{\prime}(y)=0$, we know that problem (1) has two nontrivial solutions for almost all $\lambda \in\left(1 / 2 \kappa_{1}, 1 / 2 \kappa_{0}\right)$. The proof is completed.

In order to prove Theorem 2, we need to recall some propositions of genus and the symmetric mountain pass theorem given by Kajikiya [27].

Let $E$ be a real Banach space and let $\Sigma$ denote the family of sets $A \subset E \backslash\{0\}$ such that $A$ is closed in $E$ and symmetric with respect to 0 (i.e., $x \in A \Rightarrow-x \in A$ ). For $A \in \Sigma$, we define a genus $\Upsilon(A)$ of $A$ by the smallest integer $n$ such that there exists an odd continuous mapping from $A$ to $\mathbf{R}^{n} \backslash\{0\}$. If such a $n$ does not exist, we define $\Upsilon(A)=\infty$. Moreover, set $\Upsilon(\varnothing)=0$. Let $\Gamma_{k}$ denote the subsets of $\sum$ such that $\Upsilon(A) \geq k$ for all $A \in \Gamma_{k}$.

Proposition 5 (see [18], Propositions 7.5 and 7.7]). Let $A, B \subset \sum$. Then, the following hold.

$\left(\Upsilon_{1}\right)$ If there exists an odd continuous mapping from $A$ to $B$, then $\Upsilon(A) \leq \Upsilon(B)$.

$\left(\Upsilon_{2}\right)$ If $A \subset B$, then $\Upsilon(A) \leq \Upsilon(B)$.

$\left(\Upsilon_{3}\right)$ If $A$ is compact, then $\Upsilon(A)<+\infty$ and $\Upsilon\left(N_{\varepsilon}(A)\right)=\Upsilon(A)$ for $\varepsilon>0$ small enough, where $N_{\varepsilon}(A)=\{x \in E:\|x-A\| \leq \varepsilon\}$.
$\left(\Upsilon_{4}\right)$ The n-dimensional sphere $S_{n}$ has a genus of $n+1$ by the Borsuk-Ulam theorem.

Theorem 4 (see [27], Theorem 1]). Let E be an infinitedimensional Banach space and let $\Psi \in C^{1}(E, \mathbf{R})$ satisfy

(1) $\Psi(x)$ is even, bounded from below, $\Psi(0)=0$, and $\Psi$ satisfies the (PS) condition, that is, $\left\{x_{k}\right\} \subset E$ has a convergent subsequence whenever $\left\{\Psi\left(x_{k}\right)\right\}$ is bounded and $\Psi_{\prime}\left(x_{k}\right) \longrightarrow 0$ as $k \longrightarrow \infty$.

(2) For each $k \in \mathbf{N}$, there exists an $A_{k} \in \Gamma_{k}$ such that $\sup _{x \in A_{k}} \Psi(x)<0$.

Then, $\Psi$ possesses a sequence of critical points $\left\{x_{k}\right\}$ such that $\Psi\left(x_{k}\right) \leq 0, x_{k} \neq 0$ and $\lim _{k \rightarrow \infty} x_{k}=0$.

Proof of Theorem 2. Noticing that the embedding $Z \longrightarrow L^{\infty}$ is compact, there is $\delta_{1}>0$ such that

$$
|x(t)| \leq\|x\|_{\infty} \leq \delta_{1}\|x\|_{Z}, \quad x \in Z .
$$

Consider the truncated functional

$$
\begin{aligned}
\Psi(x)= & \frac{1}{2}\left[\int_{0}^{1}\left(P(t) x^{\prime}(t), x^{\prime}(t)\right) \mathrm{d} t+\int_{0}^{1}|x|^{2} \mathrm{~d} t\right] \\
& -\phi\left(\|x\|_{Z}\right)\left[\frac{|x(1)|^{2} \gamma(\beta)+|x(0)|^{2} \gamma(\alpha)}{2}+\frac{1}{2} \int_{0}^{1}|x|^{2} \mathrm{~d} t\right. \\
& \left.-\frac{1}{2} \int_{0}^{1}(B(t) x, x) \mathrm{d} t+\lambda \int_{0}^{1} V(t, x) \mathrm{d} t\right],
\end{aligned}
$$

for all $x \in Z$, where $\phi: \mathbf{R}^{+} \longrightarrow[0,1]$ is a nonincreasing $C^{1}$ function with $\phi(t)=1$ for $0 \leq t \leq 1 / 2 \delta_{1}$ and $\phi(t)=0$ for $t \geq 1 / \delta_{1}$. Clearly, $\Psi \in C^{1}(Z, \mathbf{R})$ and $\Psi(0)=0$. Using $\left(V_{4}\right)$, we have $\Psi(-x)=\Psi(x)$ for all $x \in Z$.

Since $P(t) \in C^{1}\left([0,1], \mathscr{L}_{s}\left(\mathbf{R}^{n}\right)\right)$ is positive definite for $t \in[0,1]$, there are $0<p_{1} \leq p_{2}$ such that

$$
\begin{aligned}
\min \left\{p_{1}, 1\right\}\|x\|_{Z}^{2} & \leq p_{1} \int_{0}^{1}\left|x^{\prime}(t)\right|^{2} \mathrm{~d} t+\int_{0}^{1}|x|^{2} \mathrm{~d} t \\
& \leq \int_{0}^{1}\left(P(t) x^{\prime}(t), x^{\prime}(t)\right) \mathrm{d} t+\int_{0}^{1}|x|^{2} \mathrm{~d} t \\
& \leq p_{2} \int_{0}^{1}\left|x^{\prime}(t)\right|^{2} \mathrm{~d} t+\int_{0}^{1}|x|^{2} \mathrm{~d} t \\
& \leq \max \left\{p_{2}, 1\right\}\|x\|_{Z}^{2},
\end{aligned}
$$

for all $x \in Z$, which implies that $\left(\int_{0}^{1}\left(P(t) x^{\prime}(t), x^{\prime}(t)\right) \mathrm{d} t+\right.$ $\left.\int_{0}^{1}|x|^{2} \mathrm{~d} t\right)^{1 / 2}$ is also an equivalent norm on $Z$. Thus, for $\|x\|_{Z} \geq 1 / \delta_{1}$, we have

$$
\begin{gathered}
\Psi(x)=\frac{1}{2}\left[\int_{0}^{1}\left(P(t) x^{\prime}(t), x^{\prime}(t)\right) \mathrm{d} t+\int_{0}^{1}|x|^{2} \mathrm{~d} t\right] \\
\longrightarrow+\infty, \quad \text { as }\|x\|_{Z} \longrightarrow+\infty
\end{gathered}
$$


which shows that $\Psi$ is bounded from below and satisfies the (PS) condition.

It is known that the operator $\Lambda_{B}$ is self-adjoint and $\sigma\left(\Lambda_{B}\right)=\sigma_{d}\left(\Lambda_{B}\right)$ is bounded from below. This shows that the operator $\Lambda_{B}$ has a sequence of eigenvalues

$$
\begin{aligned}
& -\infty<\lambda_{0} \leq \lambda_{1} \leq \cdots \leq \lambda_{n_{0}} \leq 0<\lambda_{n_{0}+1} \\
& \quad \leq \lambda_{n_{0}+2} \leq \cdots \leq \lambda_{n_{0}+n} \leq \cdots \longrightarrow+\infty,
\end{aligned}
$$

and the system of eigenfunctions $\left\{e_{n}: n \in \mathbf{N}\right\}$ corresponding to $\left\{\lambda_{n}: n \in \mathbf{N}\right\}$ forming an orthogonal basis in $L^{2}=X$. Given any $k \in \mathbf{N}$, let $Z_{k}=\oplus_{j=1}^{k} X_{j}$, where $X_{j}=\operatorname{span}\left\{e_{j}\right\}$. By the equivalence of the norms on the finite-dimensional space $Z_{k}$, we know that there exists a constant $\zeta_{k}>0$ such that

$$
\zeta_{k}\|x\|_{L^{2}} \geq\|x\|_{Z}, \quad \forall x \in Z_{k} .
$$

Noticing that $B(t) \in L^{\infty}\left([0,1], \mathscr{L}_{s}\left(\mathbf{R}^{n}\right)\right)$, we know that there is $d_{1}>0$ such that

$$
\left|\int_{0}^{1}(B(t) x, x) \mathrm{d} t\right| \leq d_{1}\|x\|_{L^{2}}^{2}, \quad \forall x \in Z
$$

By Lemma 2.3.1 of [15], we can see that there is $d_{2}>0$ such that

$$
|x(0)|^{2}+|x(1)|^{2} \leq \int_{0}^{1}\left|x^{\prime}\right|^{2} \mathrm{~d} t+d_{2} \int_{0}^{1}|x|^{2} \mathrm{~d} t, \quad \forall x \in Z .
$$

Hence, fix $\alpha \in[0, \pi), \beta \in(0, \pi]$, and there is $d_{3}=\max \left\{d_{2}, 1\right\}\{|\gamma(\alpha)|+|\gamma(\beta)|+1\}>0$ such that

$$
\begin{aligned}
& \left(|x(0)|^{2}+|x(1)|^{2}\right)(|\gamma(\alpha)|+|\gamma(\beta)|) \leq d_{3}\left[\int_{0}^{1}\left|x^{\prime}\right|^{2} \mathrm{~d} t+\int_{0}^{1}|x|^{2} \mathrm{~d} t\right] \\
& =d_{3}\|x\|_{Z}^{2}, \quad \forall x \in Z .
\end{aligned}
$$

Fix $\lambda>0$; using $\left(V_{5}\right)$, there exists $d_{4}>0$ such that

$$
V(t, x) \geq \frac{\kappa_{3}}{\lambda} \zeta_{k}^{2}|x|^{2}
$$

for all $|x| \leq d_{4}$ and a.e. $t \in[0,1]$, where $\kappa_{3}=\max \left\{p_{2}, d_{1}\right\}+d_{3}$. Therefore, for $x \in Z_{k}$ with $\|x\|_{Z}=\rho_{k}:=\min \left\{1 / 2 \delta_{1}, d_{4} / \delta_{1}\right\}$, using (45)-(51) and (53) and (54), we can obtain $|x(t)| \leq d_{4}$ and

$$
\begin{aligned}
\Psi(x)=I(x)= & \frac{1}{2} \int_{0}^{1}\left(P(t) x^{\prime}(t), x^{\prime}(t)\right) \mathrm{d} t-\frac{|x(1)|^{2} \gamma(\beta)+|x(0)|^{2} \gamma(\alpha)}{2} \\
& +\frac{1}{2} \int_{0}^{1}(B(t) x, x) \mathrm{d} t-\lambda \int_{0}^{1} V(t, x) \mathrm{d} t \\
\leq & \frac{p_{2}}{2}\left\|x^{\prime}\right\|_{L^{2}}^{2}+\frac{d_{3}}{2}\|x\|_{Z}^{2}+\frac{d_{1}}{2}\|x\|_{L^{2}}^{2}-\kappa_{3} \zeta_{k}^{2} \int_{0}^{1}|x|^{2} \mathrm{~d} t \\
\leq & \frac{\kappa_{3}}{2}\|x\|_{Z}^{2}-\kappa_{3}\|x\|_{Z}^{2}=-\frac{\kappa_{3}}{2} \rho_{k}^{2},
\end{aligned}
$$

which implies that

$$
\left\{x \in Z_{k}:\|x\|_{Z}=\rho_{k}\right\} \subset\left\{x \in Z: \Psi(x) \leq-\frac{\kappa_{3}}{2} \rho_{k}^{2}\right\} .
$$

Put $A_{k}=\left\{x \in Z: \Psi(x) \leq-\left(\kappa_{3} / 2\right) \rho_{k}^{2}\right\}$; by Proposition 5, we have

$$
\Upsilon\left(A_{k}\right) \geq \Upsilon\left(\left\{x \in Z_{k}:\|x\|_{Z}=\rho_{k}\right\}\right)=k,
$$

which shows that $A_{k} \in \Gamma_{k}$ and

$$
\sup _{x \in A_{k}} \Psi(x) \leq-\frac{\kappa_{3}}{2} \rho_{k}^{2}<0 .
$$

By Theorem 4, we obtain that $\Psi$ possesses a sequence of critical points $\left\{x_{k}\right\}$ such that $\Psi\left(x_{k}\right) \leq 0, x_{k} \neq 0$ and $\lim _{k \longrightarrow \infty} x_{k}=0$. Since $\lim _{k \longrightarrow \infty} x_{k}=0$, for $1 / 2 \delta_{1}>0$, there is $N>0$ such that $\left\|x_{k}\right\|_{Z} \leq 1 / 2 \delta_{1}(\forall k>N)$. Thus, for $k>N$, the critical points of $\Psi$ are just critical points of $I$ via $\Psi(x)=$ $I(x)$ as $\|x\|_{Z} \leq 1 / 2 \delta_{1}$, which shows that problem (1) has infinitely many small-energy solutions for each $\lambda>0$. The proof is completed.

\section{Applications to Sturm-Liouville Equations and Examples}

In this section, we consider the applications of Theorems 1 and 2 to Sturm-Liouville equations with the mixed boundary value conditions and the Neumann boundary value conditions. Meanwhile, some examples of problems (3) and (4) are given to illustrate the validity of our result, and the result is also new even in the cases of (3) and (4).

As first special case, we consider the mixed boundary value problem (3):

$$
\left\{\begin{array}{l}
-\left(P(t) x^{\prime}(t)\right)^{\prime}+B(t) x(t)=\lambda f(t, x), \quad \text { a.e. } t \in[0,1] \\
x(0)=x^{\prime}(1)=0
\end{array}\right.
$$

where $\lambda>0, B(t) \in L^{\infty}([0,1], \mathbf{R})$, and $P(t) \in C^{1}([0,1], \mathbf{R})$ with $P(t)$ being positive definite for $t \in[0,1]$. In problem (1), taking $n=1, \alpha=0, \beta=\pi / 2$, the following corollary is immediately obtained from Theorem 1 . 
Corollary 1. Assume that $f(t, x):[0,1] \times \mathbf{R} \longrightarrow \mathbf{R}$ is a $L^{1}$-Carathéodory function and satisfies $\left(V_{1}\right),\left(V_{2}\right)$, and $\left(V_{3}\right)$ with $a(x, x)=\int_{0}^{1}\left(P(t) x^{\prime}, x^{\prime}\right) d t$ and $V(t, x)$ replaced with $F(t, x)=\int_{0}^{x} f(t, s) d s$. Then, for problem (3), the conclusion of Theorem 1 is still valid.

An example of Corollary 1 is given below.

Example 1. Consider the following problem:

$$
\left\{\begin{array}{l}
-x^{\prime \prime}(t)-\frac{\pi^{2}}{8} x(t)=\lambda f(t, x) \\
x(0)=x^{\prime}(1)=0
\end{array}\right.
$$

where

$$
F(t, x)=\int_{0}^{x} f(t, s) \mathrm{d} s= \begin{cases}l(t)|x|^{\vartheta}, & |x| \leq 1, \\ l(t)\left(|x|^{3}-\frac{3-\vartheta}{2}|x|^{2}+\frac{3-\vartheta}{2}\right), & |x|>1,\end{cases}
$$

where $1 \leq \vartheta<2$ and $l(t) \in C\left([0,1], \mathbf{R}^{+}\right)$with $l(t) \equiv 0$. Clearly, $f(t, x)$ is a $L^{1}$-Carathéodory function. By Remark 3, we have $v_{0, \pi / 2}^{I_{1}}\left(-\left(\pi^{2} / 8\right)\right)=i_{0, \pi / 2}^{I_{1}}\left(-\left(\pi^{2} / 8\right)\right)=0$, which implies that $\left(V_{1}\right)$ holds. Taking that $\eta=1, g(t)=$ $\max _{t \in[0,1]} l(t)=M_{2}$, then $\left(V_{2}\right)$ holds. After a simple calculation, we have $\delta_{0}=1, \lambda_{0}=\pi^{2} / 8$, and the eigenfunction of $\Lambda_{-\left(\pi^{2} / 8\right)}$ corresponding to the first eigenvalue $\lambda_{0}$ is $\varphi=\sin ((\pi / 2) t), t \in[0,1]$. Let $\kappa_{0}=\delta_{0} \eta^{9-2}\|g\|_{L^{1}}=M_{0}$. Noticing that

$$
\begin{aligned}
& \liminf _{c \longrightarrow 0} \frac{\int_{0}^{1} F(t, c \varphi) \mathrm{d} t}{c^{2}\|\varphi\|_{L^{2}}^{2}}=\liminf _{c \longrightarrow 0} \frac{\int_{0}^{1} l(t)|\varphi|^{9} \mathrm{~d} t}{c^{2-9}\|\varphi\|_{L^{2}}^{2}}=+\infty, \\
& \liminf _{c \longrightarrow+\infty} \frac{\int_{0}^{1} F(t, c \varphi) \mathrm{d} t}{c^{2}\|\varphi\|_{L^{2}}^{2}}=\liminf _{c \longrightarrow+\infty} \frac{\int_{0}^{1} l(t)\left[c^{3}|\varphi|^{3}-(3-\vartheta / 2) c^{2}|\varphi|^{2}+(3-\vartheta / 2)\right] \mathrm{d} t}{c^{2}\|\varphi\|_{L^{2}}^{2}}=+\infty,
\end{aligned}
$$

there exists a constant $\kappa_{1}>\kappa_{0}=M_{0}$ such that (6) and (7) of $\left(V_{3}\right)$ hold. Hence, $F(t, x)$ satisfies the hypotheses of Corollary 1 , and for almost all $\lambda \in\left(1 / 2 \kappa_{1}, 1 / 2 \kappa_{0}\right)$, problem (60) has two nontrivial solutions.

Remark 4. Obviously, $F(t, x)$ in Example 1 does not satisfy the condition $\int_{0}^{1 / 2} F(t, x) \mathrm{d} t>0(\forall x \in[0, d])$ of Theorem 3.1 in [1] and the condition $F(t, x) \leq \mu(t)\left(1+|x|^{s}(s<2, x \in \mathbf{R})\right.$ of Theorem 3.1 in [2]. Moreover, noticing that $B(t) \geq 0$ must be assumed in the condition of Theorem 3.1 in $[1,2,9,10]$, we easily see that Theorem 3.1 in $[1,2,9,10]$ cannot be applied to Example 1. This shows that Corollary 1 is also new even in the cases of (3).

Taking $n=1, \alpha=0, \beta=\pi / 2$, from Theorem 2 , we immediately obtain the following corollary.

Corollary 2. Assume that $f(t, x):[0,1] \times \mathbf{R} \longrightarrow \mathbf{R}$ is a $L^{1}$-Carathéodory function and satisfies $\left(V_{4}\right)$ and $\left(V_{5}\right)$ with $V(t, x)$ replaced with $F(t, x)=\int_{0}^{x} f(t, s) d s$. Then, for problem (3), the conclusion of Theorem 2 is still valid.

Example 2. Consider

$$
\left\{\begin{array}{l}
-x^{\prime \prime}(t)+b(t) x(t)=\lambda f(t, x) \\
x(0)=x^{\prime}(1)=0
\end{array}\right.
$$

where

$$
F(t, x)=\int_{0}^{x} f(t, s) \mathrm{d} s=l_{1}(t)\left(|x|^{3 / 2}+\ln (1+|x|)\right)+|x|^{3},
$$

$\lambda>0, b(t) \in L^{\infty}([0,1], \mathbf{R})$ and $l_{1}(t) \in C\left([0,1], \mathbf{R}^{+}\right)$with $\min _{t \in[0,1]}\{l(t)\}>0$. Clearly, $f(t, x)$ is a $L^{1}$-Carathéodory function, $F(t, 0)=0$, and $\left(V_{4}\right)$ holds. Since

$$
\lim _{|x| \longrightarrow 0} \frac{F(t, x)}{|x|^{2}}=\lim _{|x| \longrightarrow 0} \frac{l_{1}(t)\left(|x|^{3 / 2}+\ln (1+|x|)\right)+|x|^{3}}{|x|^{2}}=+\infty
$$

$\left(V_{5}\right)$ holds. By Corollary 2, problem (63) has infinitely many small-energy solutions for each $\lambda>0$.

Remark 5. By Remarks 2 and 3 , we know that $b(t)<0$ is also allowed. Noticing that $b(t) \geq 0$ and $\lambda \in\left(\lambda_{1}, \lambda_{2}\right) \subset(0,+\infty)$ must be assumed in the condition of Theorem 3.1 in $[3,4]$, we easily see that Theorem 3.1 in $[3,4]$ cannot be applied to Example 2. This shows that Corollary 2 is also new even in the cases of (3).

As second special case, consider the Neumann boundary value problem (4)

$$
\left\{\begin{array}{l}
-\left(P(t) x^{\prime}(t)\right)^{\prime}+B(t) x(t)=\lambda f(t, x), \text { a.e. } t \in[0,1], \\
x^{\prime}(0)=x^{\prime}(1)=0
\end{array}\right.
$$

where $\lambda>0, B(t) \in L^{\infty}([0,1], \mathbf{R})$ and $P(t) \in C^{1}([0,1], \mathbf{R})$ with $P(t)$ being positive definite for $t \in[0,1]$. In problem (1), taking $n=1, \alpha=\beta=\pi / 2$, the following corollary is immediately obtained from Theorem 1 .

Corollary 3. Assume that $f(t, x):[0,1] \times \mathbf{R} \longrightarrow \mathbf{R}$ is a $L^{1}$-Carathéodory function and satisfies $\left(V_{1}\right),\left(V_{2}\right)$, and $\left(V_{3}\right)$ with $a(x, x)=\int_{0}^{1}\left(P(t) x^{\prime}, x^{\prime}\right) d t$ and $V(t, x)$ replaced with 
$F(t, x)=\int_{0}^{x} f(t, s) d s$. Then, for problem (4), the conclusion of Theorem 1 is still valid.

Now, we give an example of Corollary 3.

Example 3. Consider the following problem:

$$
\left\{\begin{array}{l}
-x^{\prime \prime}(t)+b_{1}(t) x(t)=\lambda f(t, x), \quad \text { a.e. } t \in[0,1] \\
x^{\prime}(0)=x^{\prime}(1)=0
\end{array}\right.
$$

where $F(t, x)=\int_{0}^{x} f(t, s) \mathrm{d} s$ as defined in Example 1 and

$$
b_{1}(t)= \begin{cases}1, & t \in\left[0, \frac{1}{2}\right), \\ 4 \pi^{2}+1, & t \in\left[\frac{1}{2}, 1\right] .\end{cases}
$$

Clearly, $f(t, x)$ is a $L^{1}$-Carathéodory function. By Remark 2, we have $i_{\pi / 2, \pi / 2}^{I_{1}}(1 / 2)=0$. From $b_{1}(t)>1 / 2$ and Proposition 4, we know that $i_{\pi / 2, \pi / 2}^{I_{1}}(1 / 2) \geq v_{\pi / 2, \pi / 2}^{I_{1}}$ $\left(b_{1}(t)\right)+i_{\pi / 2, \pi / 2}^{I_{1}}\left(b_{1}(t)\right) \geq 0$, which implies that $\left(V_{1}\right)$ holds. Taking that $\eta=1, g(t)=\max _{t \in[0,1]} l(t)=M_{2}$, then $\left(V_{2}\right)$ holds. After a simple calculation, we have $\delta_{0}=1, \lambda_{0}=4 \pi^{2}+$ 1 and the eigenfunction of $\Lambda_{b_{1}(t)}$ corresponding to the first eigenvalue $\lambda_{0}$ is $\varphi_{0}=\left\{\begin{array}{ll}\cos 2 \pi t, & t \in[0,(1 / 2)), \\ -1, & t \in[(1 / 2), 1] .\end{array}\right.$ Let $\kappa_{0}=\delta_{0} \eta^{9-2}\|g\|_{L^{1}}=M_{0}$. Noticing that

$$
\begin{array}{r}
\liminf _{c \longrightarrow 0} \frac{\int_{0}^{1} l(t)\left|\varphi_{0}\right|^{9} \mathrm{~d} t}{c^{2-9}\left\|\varphi_{0}\right\|_{L^{2}}^{2}}=+\infty, \\
\liminf _{c \longrightarrow+\infty} \frac{\int_{0}^{1} l(t)\left[c^{3}\left|\varphi_{0}\right|^{3}-((3-9) / 2) c^{2}\left|\varphi_{0}\right|^{2}+(3-9) / 2\right] \mathrm{d} t}{c^{2}\left\|\varphi_{0}\right\|_{L^{2}}^{2}}=+\infty,
\end{array}
$$

then there exists a constant $\kappa_{1}>\kappa_{0}=M_{0}$ such that (6) and (7) of $\left(V_{3}\right)$ hold. Hence, $F(t, x)$ satisfies the hypotheses of Corollary 3 , and for almost all $\lambda \in\left(1 / 2 \kappa_{1}, 1 / 2 \kappa_{0}\right)$, problem (67) has two nontrivial solutions.

Remark 6. Noticing that $B(t) \equiv M \geq 0$ and $\lambda=1$ must be assumed in the condition of Theorem 1 in $[11,12]$, we easily see that Theorem 1 in $[11,12]$ cannot be applied to Example 3. This shows that Corollary 3 is also new even in the cases of (4).

Taking $n=1, \alpha=\beta=\pi / 2$, from Theorem 2, we have the following corollary.

Corollary 4. Assume that $f(t, x):[0,1] \times \mathbf{R} \longrightarrow \mathbf{R}$ is a $L^{1}$-Carathéodory function and satisfies $\left(V_{4}\right)$ and $\left(V_{5}\right)$ with $V(t, x)$ replaced with $F(t, x)=\int_{0}^{x} f(t, s) d s$. Then, for problem (4), the conclusion of Theorem 2 is still valid.

Example 4. Finally, consider

$$
\left\{\begin{array}{l}
-x^{\prime \prime}(t)+b(t) x(t)=\lambda f(t, x) \\
x^{\prime}(0)=x^{\prime}(1)=0
\end{array}\right.
$$

where $F(t, x)=\int_{0}^{x} f(t, s) \mathrm{d} s$ as defined in Example 2, $\lambda>0$, and $b(t) \in L^{\infty}([0,1], \mathbf{R})$. Clearly, $f(t, x)$ is a $L^{1}$-Carathéodory function, $F(t, 0)=0$, and $\left(V_{4}\right)$ and $\left(V_{5}\right)$ hold. By Corollary 4 , problem (70) has infinitely many small-energy solutions for each $\lambda>0$.

Remark 7. By Remarks 2 and 3, we know that $b(t)<0$ is also allowed. Noticing that $b(t) \in C\left([0,1], \mathbf{R}^{+} \backslash\{0\}\right)$ and $\lambda \in\left(\lambda_{1}, \lambda_{2}\right) \subset(0,+\infty)$ must be assumed in the condition of
Theorem 3.1 in [5], we easily see that Theorem 3.1 in [5] cannot be applied to Example 4 . This implies that Corollary 4 is also new even in the cases of (4).

\section{Data Availability}

No data were used to support this study.

\section{Conflicts of Interest}

The authors declare that they have no conflicts of interest.

\section{Authors' Contributions}

All authors typed, read, and approved the final manuscript.

\section{Acknowledgments}

This research was supported by the National Natural Science Foundation of China (no. 11901248).

\section{References}

[1] D. Averna, N. Giovannelli, and E. Tornatore, "Existence of three solutions for a mixed boundary value problem with the Sturm-Liouville equation," Bulletin of the Korean Mathematical Society, vol. 49, no. 6, pp. 1213-1222, 2012.

[2] D. Averna and R. Salvati, "Three solutions for a mixed boundary value problem involving the one-dimensional $p$ Laplacian," Journal of Mathematical Analysis and Applications, vol. 298, pp. 245-260, 2004.

[3] G. A. Afrouzi, A. Hadjian, and S. Heidarkhani, "Infinitely many solutions for a mixed doubly eigenvalue boundary value 
problem," Mediterranean Journal of Mathematics, vol. 10, pp. 1317-1331, 2013.

[4] G. Bonanno and E. Tornatore, "Infinitely many solutions for a mixed boundary value problem," Annales Polonici Mathematici, vol. 99, pp. 285-293, 2010.

[5] G. Bonanno and G. D'Agui, "A Neumann boundary value problem for the Sturm-Liouville equation," Applied Mathematics and Computation, vol. 208, pp. 318-327, 2009.

[6] G. Bonanno and G. D'Agui, "Two non-zero solutions for elliptic Dirichlet problems," Zeitschrift für Analysis und ihre Anwendungen, vol. 35, pp. 449-464, 2016.

[7] G. Bonanno, A. Iannizzotto, and M. Marras, "Two positive solutions for superlinear Neumann problems with a complete Sturm-Liouville operator," Journal of Convex Analysis, vol. 25, pp. 421-434, 2018.

[8] G. D'Aguì, "Existence results for a mixed boundary value problem with Sturm-Liouville equation," Advances in Pure and Applied Mathematics, vol. 2, pp. 237-248, 2011.

[9] G. D’Aguì, A. Sciammetta, and E. Tornatore, "Two non-zero solutions for Sturm-Liouville equations with mixed boundary conditions," Nonlinear Analysis Real World Applications, vol. 47, pp. 324-331, 2019.

[10] A. Hadjian and S. Tersian, "Existence results for a mixed boundary value problem," Electronic Journal of Qualitative Theory of Differential Equations, vol. 78, pp. 1-15, 2015.

[11] J. P. Sun and W. T. Li, "Multiple positive solutions to secondorder Neumann boundary value problems," Applied Mathematics and Computation, vol. 146, pp. 187-194, 2003.

[12] J. P. Sun, W. T. Li, and S. S. Cheng, "Three positive solutions for second-order Neumann boundary value problems," $A p$ plied Mathematics Letters, vol. 17, pp. 1079-1084, 2004.

[13] G. Bonanno and P. Candito, "On differentiable functionals and applications to elliptic problems with discontinuous nonlinearities," Journal of Differential Equations, vol. 244, pp. 3031-3059, 2008.

[14] B. Ricceri, "A general variational principle and some of its applications," Journal of Computational and Applied Mathematics, vol. 133, pp. 401-410, 2000.

[15] Y. J. Dong, "Index theory for linear self-adjoint operator equations and nontrivial solutions for asymptotically linear operator equations," Calculus of Variations and Partial Differential Equations, vol. 38, pp. 75-109, 2010.

[16] Y. J. Dong, Index Theory for Hamiltonian Systems and Multiple Solutions Problems, Science Press, Beijing, China, 2014.

[17] J. Mawhin and M. Willem, Critical Point Theory and Hamiltonian Systems, Springer-Verlag, New York, NY, USA, 1989.

[18] P. H. Rabinowitz, "Minimax methods in critical point theory with applications to differential equations," CBMS Regional Conference Series in Mathematics, vol. 65, 1986.

[19] M. Schechter, Minmax Systems and Critical Point Theory, Birkhäuser, Boston, MA, USA, 2009.

[20] M. Schechter, "Nonautonomous second order Hamiltonian systems," Pacific Journal of Mathematics, vol. 251, no. 2, pp. 431-452, 2011.

[21] Y. Y. Ye and C. L. Tang, "Infinitely many periodic solutions of non-autonomous second-order Hamiltonian systems," SAVE Proceedings, vol. 144A, pp. 205-223, 2014.

[22] W. Zou and S. Li, "Infinitely many solutions for Hamiltonian systems," Journal of Differential Equations, vol. 186, pp. 141-164, 2002.

[23] Q. Zhang and C. Liu, "Infinitely many periodic solutions for second order Hamiltonian systems," Journal of Differential Equations, vol. 251, pp. 816-833, 2011.
[24] G. Bonanno, R. Livrea, and M. Schechter, "Multiple solutions of second order Hamiltonian systems," Electronic Journal of Qualitative Theory of Differential Equations, vol. 33, pp. 1-15, 2017.

[25] G. Fei, "Multiple solutions of some nonlinear strongly resonant elliptic equations without the (PS) condition," Journal of Mathematical Analysis and Applications, vol. 193, pp. 659-670, 1995.

[26] W. Zou, "Variant fountain theorems and their applications," Manuscripta Mathematica, vol. 104, pp. 343-358, 2001.

[27] R. Kajikiya, "A critical point theorem related to the symmetric mountain pass lemma and its applications to elliptic equations," Journal of Functional Analysis, vol. 225, pp. 352-370, 2005.

[28] Y. Chen, Y. Dong, and Y. Shan, "Existence of solutions for sublinear or superlinear operator equations," Science China Mathematics, vol. 58, pp. 1653-1664, 2015.

[29] M. L. Song, "Existence of solutions for subquadratic convex or $B$-concave operator equations and applications to second order Hamiltonian systems," Electronic Journal of Qualitative Theory of Differential Equations, vol. 49, pp. 1-19, 2020. 